\title{
CNS Involvement in Acute Posterior Multifocal Placoid Pigment Epitheliopathy
}

\author{
Christian Pagnoux, Carter Thorne, Efrem D. Mandelcorn, Simon Carette
}

Can. J. Neurol. Sci. 2011; 38: 526-528

Acute posterior multifocal placoid pigment epitheliopathy (APMPPE) is a rare disease of the choroid and retina, of unknown etiology and mostly affecting young subjects, that can involve the central nervous system (CNS) due to possible associated vasculitis or granulomatous inflammation. Central nervous system manifestations can range from headache, to meningo-encephalitis, to stroke $\mathrm{e}^{1,2}$. Less than 30 cases of APMPPE with CNS involvement have been reported in the literature so far. We report herein the case of a woman in her early 20's who developed CNS manifestations within a month following the diagnosis of APMPPE.

\section{Case Report}

A 22-year-old student, with no significant medical history, began complaining of diffuse headaches, bilateral floaters, and blurry vision. She was reviewed after two weeks by an ophthalmologist, who found features suggestive of APMPPE (Figure A-C). The magnetic resonance image (MRI) of her brain was normal, and no treatment was recommended. However, her headaches worsened, and two weeks later she began experiencing fluctuating numbness in her limbs. She started prednisone at a dose of $60 \mathrm{mg} / \mathrm{d}$ with a progressive tapering schedule, which led to a minor improvement in her symptoms.

Two months later, while she was still on a dose of $20 \mathrm{mg} / \mathrm{d}$ of prednisone, she developed sudden-onset left hemiparesis, tingling in her limbs, and mild behavioural changes. She was admitted to hospital. Laboratory investigations showed normal erythrocyte sedimentation rate (ESR, $23 \mathrm{~mm}$ in first hour), Creactive protein (CRP, $8 \mathrm{mg} / \mathrm{l})$, and creatinine level $(63 \mu \mathrm{mol} / \mathrm{l})$. Urinalysis, chest X-ray, and brain computed tomogram (CT) were normal. Cerebral spinal fluid (CSF) examination showed an elevated white cell count at $14 / \mathrm{mm}^{3}$ (mainly lymphocytes), a red cell count at $20 / \mathrm{mm}^{3}$, and a protein level of $0.44 \mathrm{~g} / \mathrm{l}(\mathrm{N}<0.60 \mathrm{~g} / \mathrm{l})$. She was prescribed IV vancomycin, co-trimoxazole, and acyclovir. Cerebral spinal fluid culture and viral studies by polymerase chain reaction were negative for cytomegalovirus, enterovirus, herpes, and varicella-zoster viruses, so these medications were stopped after one week. She was also treated with $1 \mathrm{~g}$ of methyprednisolone daily for four days, followed by oral prednisone $(60 \mathrm{mg} / \mathrm{d})$. Brain MRI on Day 2 post-admission showed multiple lesions in the right cervical medulla and left pons and in the left parietal lobe (Figure D-F). Brain angio-MRI showed no abnormality, and MRI of the thoracic and lumbar spinal cord was also normal. Additional tests revealed slightly elevated polyclonal serum gammaglobulins (31 g/l), speckled and homogeneous antinuclear antibodies (1/160), without specificity, and normal complement. Antineutrophil cytoplasmic

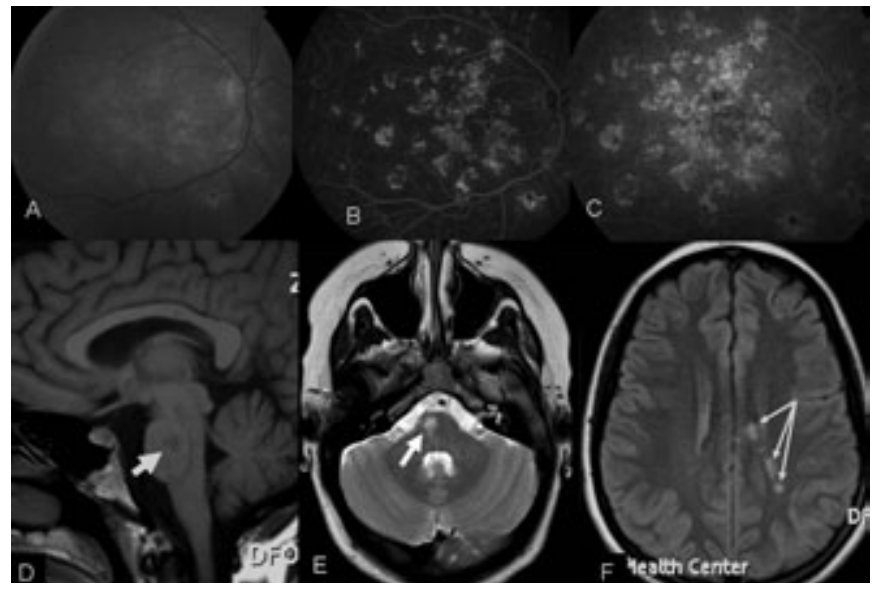

Figure : Eye examination and cerebral MRI. (A) Eye examination of the retina showing multiple yellow-white placoid lesions primarily in the posterior fundus, demonstrating $(B)$ early hypofluorescence with $(C)$ late staining on fluorescein angiography. (D) MRI of the brain showing hypointense pons lesion on T1 sequence, $(E)$ hyperintense lesion of the right cervical medulla on T2 sequence, and $(F)$ supra-ventricular hyperintense lesions on T2-FLAIR sequences.

antibodies, rheumatoid factor, antiphospholipid and anticardiolipin antibodies, as well as serologies for borreliosis, syphilis, human immunodeficiency virus (HIV), and hepatitis B and $\mathrm{C}$ were all negative. Serum angiotensin-converting enzyme level was normal, and tuberculin skin test was negative.

She recovered partially but, two weeks later, she still had dysesthesia and partial motor deficit of her left foot, with extensor plantar response and left side hyperreflexia. A repeat lumbar puncture was normal, and a repeat brain MRI showed a slight regression of the left upper pons lesion. When compared with the initial brain MRI, diffusion and apparent diffusion coefficient (ADC) sequences were further in favour of ischemic rather than demyelinating lesions. There was no change in the

From the Division of Rheumatology (CP, SC) and Department of Ophthalmology (EDM), Mount Sinai Hospital/University Health Network, Toronto; Southlake Regional Health Centre (CT), Newmarket, Ontario, Canada.

Received November 29, 2010. Final Revisions Submitted January 10, 2011. Correspondence to: Christian Pagnoux, Mount Sinai Hospital/University Health Network, Division of Rheumatology, The Rebecca MacDonald Centre for Arthritis and Autoimmunity, 60 Murray Street, Ste 2-001, Toronto, Ontario, M5T 3L9, Canada. 
other lesions. Azathioprine was added to her medication. Six months later (i.e. nine and a half months after the onset of her first visual symptoms), she had no remaining floaters, no complaints of blurry vision, nor any neurological symptoms or sensory-motor deficits. Retinal pigmental scars were noticed on fundus examination, but not directly affecting the central macular areas.

\section{Discussion}

Acute posterior multifocal placoid pigment epitheliopathy is a rare acute chorioretinal inflammatory disease of unknown etiology, characterized by the sudden appearance of yellowwhite placoid lesions at the level of the retinal pigment epithelium and the choriocapillaris. The disease is seen mostly in young patients (mean age around 25 years [range, 7-51]), has an equal gender distribution, and affects both eyes in $>85 \%$ of cases $^{1,2}$. Visual symptoms include blurred vision, scotomas, metamorphopsia, and floaters. Retinal examination reveals multiple yellowish retinal placoid spots within the macular region that may extend beyond the retinal arcades. Fluorescein angiography of these lesions classically demonstrates early hypofluorescence with late staining, as was seen in our patient. Additional non-invasive ocular imaging modalities include optical coherence tomography (OCT) and fundus autofluorescence $^{3}$. Optical coherence tomography may demonstrate shallow neurosensory retinal detachment during acute phase of APMPPE with focal areas of hyper-reflectance corresponding to outer retinal inflammatory deposits. Fundus autofluorescence is helpful to distinguish acute (decreased autofluorescence) from healed lesions (increased autofluorescence).

Retinal lesions of APMPPE are believed to result from ischemic changes, possibly secondary to vasculitis of the small choroidal arterioles. The precise pathophysiologic mechanisms of APMPPE, however, remain unknown. Some studies have suggested a post-infectious etiology since $40 \%$ of patients diagnosed with APMPPE report flu-like symptoms prior to the onset of visual symptoms; adenoviral or streptococcal infections have been documented in some of these cases ${ }^{1,2}$. Acute posterior multifocal placoid pigment epitheliopathy following vaccinations against meningococcus $\mathrm{C}$, influenza, or hepatitis $\mathrm{B}$ viruses have also been reported, suggesting an immune-mediated mechanism, rather than a direct effect of the infectious agent ${ }^{4}$.

To date, up to 30 patients with APMPPE-associated CNS involvement have been reported, with a slight male preponderance $e^{2,5,6}$. The CNS manifestations range from headaches, to encephalopathy, to (rarely) fatal cerebral infarcts. Most reported patients with CNS manifestations had stroke-like manifestations. The interval between the onset of visual disturbances and CNS manifestations is usually $<1$ month, but may be as long as six months. Brain CT scan and MRI may be initially normal. When neurological involvement worsens, MRI can reveal ischemic and/or hemorrhagic infarcts, often in the occipital areas, but lesions in the pons, periventricular region, and corpus callosum have also been reported. Cerebral venous thrombosis is exceptional ${ }^{2}$. Cerebral magnetic resonance angiography rarely reveals arterial abnormalities, possibly because the involved vessels are too small for the resolution capacity of this technique. Conventional angiography may be more sensitive, but it is rarely performed in these patients ${ }^{1,2}$.
Cerebral spinal fluid examination demonstrates pleocytosis and/or elevated protein levels in $>80 \%$ of patients ${ }^{1,2}$. There is usually no systemic inflammatory syndrome, or only slightly elevated CRP and/or ESR.

Granulomatous vasculitis and/or granulomatous inflammation are the two main pathological findings in the rare cases of APMPPE-related CNS disease for which tissue has been obtained by brain autopsy ${ }^{5}$. One report has revealed granulomas with multinucleated giant cells (but no vasculitis) in the choroid, near the retinal pigment epithelium, as well as, intriguingly, in the liver, heart, spleen, and lungs in one patient ${ }^{5}$. In another report, nongranulomatous vasculitis was shown on muscle biopsy $^{7}$. Interestingly, APMPPE has been reported in association with other granulomatous diseases, including Wegener's granulomatosis, sarcoidosis, and pulmonary tuberculosis.

Eye lesions of APMPPE usually heal spontaneously within weeks, but leave retinal scars, possibly leading to persistent visual abnormalities, particularly in patients with foveal involvement $(60-70 \% \text { of patients })^{1,2}$. Thus, some ophthalmologists advocate systemic corticosteroids with early foveal involvement (starting with $0.5-1 \mathrm{mg} / \mathrm{kg} / \mathrm{d}$ of prednisone, with a subsequent and slow dose tapering to reach a daily dose around 5-20 $\mathrm{mg}$ at Month 3, and $5 \mathrm{mg} / \mathrm{d}$ at Month 6). When the CNS is involved, most authors recommend the prompt use of high-dose corticosteroids (IV methylprednisolone pulse(s) $7.5-15 \mathrm{mg} / \mathrm{kg} / \mathrm{d}$ for one to three days for severe cases, followed by prednisone 1 $\mathrm{mg} / \mathrm{kg} / \mathrm{d}$ for two to four weeks, with a subsequent tapering, as described above), usually combined with an immunosuppressant agent, such as azathioprine $(2 \mathrm{mg} / \mathrm{kg} / \mathrm{d})$ or cyclophosphamide in more severe cases (IV pulses at 500-700 mg/m2 on Days 1, 14, and 28 , then every three weeks; or orally at $2 \mathrm{mg} / \mathrm{kg} / \mathrm{d}$ if IV is not possible; for a total of six pulses or three months, respectively; then switch to azathioprine, at $2 \mathrm{mg} / \mathrm{kg} / \mathrm{d}$ ). Mitoxantrone has been successfully used in a recently published case ${ }^{8}$. There is no data on methotrexate or mycophenolate mofetil as alternatives. The optimal duration of therapy is unknown, but should be at least six months, with a low maintenance dose of prednisone (around $5 \mathrm{mg} / \mathrm{d}$ ) and azathioprine for probably $\geq 12$ months. Relapsing or worsening of APMPPE and/or CNS complications is relatively rare but can occur, especially during the corticosteroid tapering period, as in five previously reported patients ${ }^{6}$. Hence, these patients should be best managed and followed by a multidisciplinary team, including ophthalmologists, neurologists and rheumatologists.

\section{ACKNOWLEDGEMENT}

The authors thank Drs. M.S. Aubrey and S. Baryshnik for their help in the management of the patient, and Dr. A. Brézin for his advice on this case.

\section{Disclosure}

Dr. Pagnoux is currently supported by grants from the Vasculitis Clinical Research Consortium and the Toronto General Hospital/Toronto Western Hospital Foundation/ University Health Network, Toronto, ON, Canada. 


\section{REFERENCES}

1. Fiore T, Iaccheri B, Androudi S, et al. Acute posterior multifocal placoid pigment epitheliopathy: outcome and visual prognosis. Retina. 2009;29:994-1001.

2. O'Halloran HS, Berger JR, Lee WB, et al. Acute multifocal placoid pigment epitheliopathy and central nervous system involvement: nine new cases and a review of the literature. Ophthalmology. 2001;108:861-8.

3. Souka AA, Hillenkamp J, Gora F, Gabel V-P, Framme C. Correlation between optical coherence tomography and autofluorescence in acute posterior multifocal placoid pigment epitheliopathy. Graefes Arch Clin Exp Ophthalmol. 2006;244: 1219-23.

4. Mendrinos E, Baglivo E. Acute posterior multifocal placoid pigment epitheliopathy following influenza vaccination. Eye (London). 2010;24:180-1.
5. de Vries JJ, den Dunnen WF, Timmerman EA, Kruithof IG, De Keyser J. Acute posterior multifocal placoid pigment epitheliopathy with cerebral vasculitis: a multisystem granulomatous disease. Arch Ophthalmol. 2006;124:910-3.

6. Luneau K, Newman NJ, Srivastava S, Biousse V. A case of acute posterior multifocal placoid pigment epitheliopathy with recurrent stroke. J Neuroophthalmol. 2009;29:111-8.

7. Bewermeyer H, Nelles G, Huber M, Althaus C, Neuen-Jacob E, Assheuer J. Pontine infarction in acute posterior multifocal placoid pigment epitheliopathy. J Neurol. 1993;241:22-6.

8. Masse H, Guyomard JL, Baudet D, Pinel JF, Edan G, Charlin JF, Mitoxantrone therapy for acute posterior multifocal placoid pigment epitheliopathy with cerebral vasculitis. Case Report Med. 2009;2009:481512. Epub 2009 May 19. 